\title{
Anthocyanins in the black soybean (Glycine max L.) protect U2OS cells from apoptosis by inducing autophagy via the activation of adenosyl monophosphate-dependent protein kinase
}

\author{
YUN-JEONG CHOE ${ }^{1}$, TAE JOUNG HA ${ }^{3}$, KYOUNG-WON KO ${ }^{1}$, \\ SUN-YOUNG LEE ${ }^{1}$, SEOK JOON SHIN ${ }^{2}$ and HO-SHIK KIM ${ }^{1}$
}

\begin{abstract}
Departments of ${ }^{1}$ Biochemistry and ${ }^{2}$ Internal Medicine, College of Medicine, The Catholic University of Korea, Seoul 137-701; ${ }^{3}$ Department of Functional Crop, National Institute of Crop Science (NCIS), Rural Development Administration (RDA), Miryang 627-803, Republic of Korea
\end{abstract}

Received June 21, 2012; Accepted July 23, 2012

DOI: 10.3892/or.2012.2034

\begin{abstract}
Anthocyanins (ATCs) have been reported to induce apoptosis in various types of cancer cells, stimulating the development of ATCs as a cancer chemotherapeutic or chemopreventive agent. It was recently reported that ATCs can induce autophagy, however, the mechanism for this remains unclear. In the present report, we carried out mechanistic studies of the mechanism involved in ATC-induced autophagy using ATCs extracted from black soybeans (cv. Cheongja 3, Glycine $\max$ L.). ATCs clearly induced hallmarks of autophagy, including LC3 puncta formation and the conversion of LC3-I to LC3-II in U2OS human osteosarcoma cells. The induction of autophagy was accompanied by the phosphorylation of multiple protein kinases including extracellular signal-regulated kinase (ERK)1/2, p38 mitogen-activated protein kinase (MAPK), c-Jun N-terminal kinase (JNK), protein kinase B (AKT) and adenosyl monophosphate-dependent protein kinase (AMPK). While chemical inhibitors against ERK1/2, p38 MAPK, JNK and AKT failed to inhibit ATC-induced autophagy, the suppres-
\end{abstract}

Correspondence to: Professor Ho-Shik Kim, Department of Biochemistry, College of Medicine, The Catholic University of Korea, 505 Banpo-dong, Socho-gu, Seoul 137-701, Republic of Korea E-mail:hoshik@catholic.ac.kr

Abbreviations: AKT, protein kinase B; AMPK, adenosyl monophosphate-dependent protein kinase; ATCs, anthocyanins; CaMKK, calmodulin-dependent protein kinase kinase; $\mathrm{CC}$, compound $\mathrm{C}$; $\mathrm{COX}$, cyclooxygenase; ERK, extracellular signal-regulated kinase; FOXO3a, forkhead box O3A; JNK, c-Jun N-terminal kinase; LC3, light chain 3; LKB1, liver kinase B1; MAPK, mitogen-activated protein kinase; mTOR, mammalian target of rapamycin; NF-kB, nuclear factor of $\kappa$ light polypeptide gene enhancer in activated $\mathrm{B}$ cells; $\mathrm{PGE}_{2}$, prostaglandin E2; PI3K, phosphoinositide 3-kinase; TAK1, transforming growth factor- $\beta$-activated kinase 1 ; ULK1, a serine/ threonine kinase with homology to yeast atg1

Key words: anthocyanins, adenosyl monophosphate-dependent protein kinase, autophagy, apoptosis, forkhead box O3A, p2 $7^{\mathrm{KIP1}}$ sion of AMPK by compound $\mathrm{C}$ (CC) as well as siRNA against $A M P K$ reduced ATC-induced autophagy. The treatment of ATCs resulted in a decrease in intracellular ATP contents and the activation of AMPK by AICAR treatment also induced autophagy. It is noteworthy that the reduction of autophagy via the inhibition of AMPK resulted in enhanced apoptosis in ATC-treated cells. In addition, siRNA against forkhead box O3A (FOXO3a), a downstream target of AMPK, suppressed ATC-induced autophagy and $p 27^{K I P I}$ siRNA increased apoptosis in ATC-treated cells. Collectively, it can be concluded that ATCs induce autophagy in U2OS cells via activation of the AMPK-FOXO3a pathway and protect cells from ATC-induced apoptosis via the AMPK-p27 ${ }^{\mathrm{KIP} 1}$ pathway. These results also suggest that autophagy-modulating agents could contribute to the efficient development of ATCs as anticancer therapy.

\section{Introduction}

Anthocyanins (ATCs) are natural phytochemicals which are produced by fruits and vegetables. ATCs have noteworthy biological functions, including anti-inflammatory and apoptosis-inducing effects. It has been reported that ATCs induce apoptosis in various cancer cell lines including hepatoma, colorectal carcinoma, gastric adenocarcinoma, leukemia and prostate cancer (1-5). The ATC-induced apoptosis occurs via both caspase-dependent and -independent mechanisms. It has been demonstrated that ATCs initially induced an alteration of protein kinase $\mathrm{B}$ (AKT) and mitogen-activated protein kinases (MAPKs) such as p38 MAPK, extracellular signal-regulated kinase (ERK)1/2 and c-Jun N-terminal kinase (JNK), which led to the upregulation of the BAX/BCL2 ratio and hence the activation of intrinsic apoptosis in cancer cells (1-4). However, in prostate cancer PC3 cells, ATCs induced caspase-independent apoptosis via the nuclear translocation of endonuclease $G$ and AIF (5). Regarding their anti-inflammatory activity, ATCs prevent the LPS- and UVB-induced expression of inflammatory mediators such as cyclooxygenase (COX)-2 and prostaglandin $\mathrm{E} 2\left(\mathrm{PGE}_{2}\right)$ by suppressing the nuclear factor of $\kappa$ light polypeptide gene enhancer in activated $\mathrm{B}$ cells $(\mathrm{NF}-\kappa \mathrm{B})$ and phosphoinositide 3-kinase (PI3K)/AKT pathways in 
human keratinocytes and macrophage cell lines $(6,7)$. This anti-inflammatory activity of ATCs was shown to be effective in vivo to subside circulatory and multiple organ failures due to endotoxemia (8). Based on these apoptosis-inducing and anti-inflammatory as well as anti-invasive and anti-angiogenic effects $(9,10)$, attempts have been made to develop ATCs as a chemopreventive agent, and promising results of pilot studies in rodent models of esophageal and colorectal cancers and human colorectal cancer patients have provided support for this hypothesis $(11,12)$.

In addition to inducing apoptosis, it has been suggested that ATCs induce autophagy in cancer and glial cells $(13,14)$, although the mechanism for this remains to be clarified. Autophagy is considered to be a cellular defense system that can protect cancer cells from anticancer therapeutics. Accordingly, autophagy inhibition augments ATC-induced apoptosis (13). A more detailed understanding of the mechanism underlying ATC-induced autophagy could contribute to the development of effective strategies for the use of ATCs as a chemopreventive or chemotherapeutic agent against cancer in the future.

AMPK, which plays a central role in energy balance and metabolism, is one of the critical regulators of autophagy induction. AMPK becomes activated once it is phosphorylated by several kinases $(15,16)$. Under metabolic stresses that restrain ATP synthesis, elevated AMP binds to the $\gamma$ subunit of AMPK for both allosteric activation of AMPK and prevention of the dephosphorylation of AMPK, leading to a sustained activity of AMPK. Allosterically activated AMPK is phosphorylated by liver kinase B1 (LKB1) at Thr172 of the $\alpha$ subunit to become fully activated. In response to elevated intracellular calcium levels and cytokine signaling, calmodulin-dependent protein kinase kinase (CaMKK) $\beta$ and transforming growth factor- $\beta$ activated kinase 1 (TAK1), respectively, phosphorylate AMPK. Activated AMPK phosphorylates mTORC1, an inhibitor of autophagy, and a serine/threonine kinase with homology to yeast atg1 (ULK1), an initiator of autophagy, thereby leading to autophagy induction $(17,18)$. $\mathrm{P} 27^{\mathrm{KIPl}}$, a CDK inhibitor, is directly phosphorylated at Thr198 and stabilized by AMPK for the induction of autophagy and, notably, the knockdown of $\mathrm{p} 27^{\mathrm{KIP} 1}$ in the presence of activated AMPK induced apoptosis instead of autophagy, an observation that explains the inhibitory effect of apoptosis by autophagy and the possibility that $\mathrm{p} 27^{\mathrm{KIP} 1}$ may mediate the decision between apoptosis and autophagy (19). Forkhead box O3a (FOXO3a), an important regulator of glucose metabolism and tumor suppression, was reported to be phosphorylated by AMPK (20). Recently, FOXO3a was shown to upregulate $\mathrm{p} 27^{\mathrm{KIPl}}$ to induce cell cycle arrest in glioma cells $(21,22)$. Therefore, it can be argued that under metabolic stress, autophagy formation can be induced through interactions among AMPK, FOXO3a, and p27 $7^{\mathrm{KIP}}$.

ATC was recently reported to suppress IGF-I-induced mammalian target of rapamycin (mTOR) phosphorylation by AMPK activation in colon cancer cells, providing further support for its anticancer activity (23). However, the relationship between autophagy and AMPK in ATC-treated cells is not yet fully understood. To address this issue, we used ATCs extracted from black soybeans (cv Cheongja 3). These ATC extracts were shown to promote apoptosis in a model of prostate hyperplasia in vivo and to induce autophagy in glial cells, which proved to contain biologically active components of autophagy induction as well as apoptosis $(14,24)$. In the present study, we observed the induction of autophagy and AMPK activation by ATCs and analyzed the role of AMPK in this autophagy.

\section{Materials and methods}

Chemicals. AICAR, compound C (CC), SP600125, SB203580, U0126, and triciribine were obtained from Sigma-Aldrich Corp. (St. Louis, MO, USA) or Merck (Darmstadt, Germany). All chemicals used are of molecular biology or cell culture grade.

Extraction of ATCs. ATCs were extracted from black seed coated soybean, Cheongja 3, developed by the National Institute of Crop Science, Rural Development Administration at Miryang, Korea, as previously described, with minor modifications (25). Briefly, hand-peeled seed coats were extracted twice with $80 \%$ ethanol containing $0.1 \%$ acetic acid for 2 days at $4^{\circ} \mathrm{C}$. These ethanol extracts were filtered through a $0.45-\mu \mathrm{m}$ filter and concentrated in a rotary evaporator to obtain crude ATC extracts. The crude extracts were freeze-dried and kept below $-70^{\circ} \mathrm{C}$.

Establishment of U2OS-GFP-LC3 cells. U2OS cells were cultured in high glucose DMEM (Invitrogen, Carlsbad, CA, USA) supplemented with $10 \%$ heat-inactivated fetal bovine serum (FBS; Hyclone Laboratories Inc., Logan, UT, USA), $100 \mathrm{U} / \mathrm{ml}$ penicillin/streptomycin (Hyclone). Plasmid EGFPLC3 was generously donated by Professor S.H. Lee at the Department of Pathology, the Catholic University of Korea. To establish stable cell lines expressing GFP-LC3, pEGFPempty vector or pEGFP-LC3 was transfected into U2OS cells. Following the selection of transfected cells using G418 for 14 days, viable cells were pooled, and GFP expression was observed under a fluorescence microscope. Total cellular extracts were prepared and subjected to immunoblot analysis against GFP or LC3.

Immunoblot analysis. U2OS cells treated with ATCs with or without other chemicals as indicated were lysed with RIPA buffer (50 mM Tris-HCl, pH 7.4, $150 \mathrm{mM} \mathrm{NaCl}, 0.25 \%$ Na-deoxycholate, $1 \%$ NP-40, 1 mM EDTA) containing protease inhibitors (Roche Applied Sciences, Indianapolis, IN, USA). Total cellular proteins were separated by SDS-polyacrylamide gels and transferred to nitrocellulose membranes (Millipore, Billerica, MA, USA), which were hybridized with primary and then secondary antibodies. Finally, protein bands were detected by enhanced chemiluminescence (ECL; GE Healthcare, UK). Antibodies against phospho-AMPK-Thr172, phospho-p38 MAPK (T180/Y182), p38 MAPK, JNK, Erk1/2, phospho-Erk1/2 (T202/Y204), mTOR, and phospho-mTOR (S2448) were obtained from Cell Signaling Technology, Inc. (Danvers, MA, USA) and antibodies against phospho-JNK (Y185/Y223), AKT, phospho-AKT (S473), ACC, phospho-ACC (S79), and cleaved PARP were from Epitomics, Inc. (Burlingame, CA, USA). Antibodies against actin, $\alpha$-tubulin, and GAPDH from Santa Cruz Biotechnology, Inc. (Santa Cruz, CA, USA) and LC3 from Sigma-Aldrich Corp. were used. 

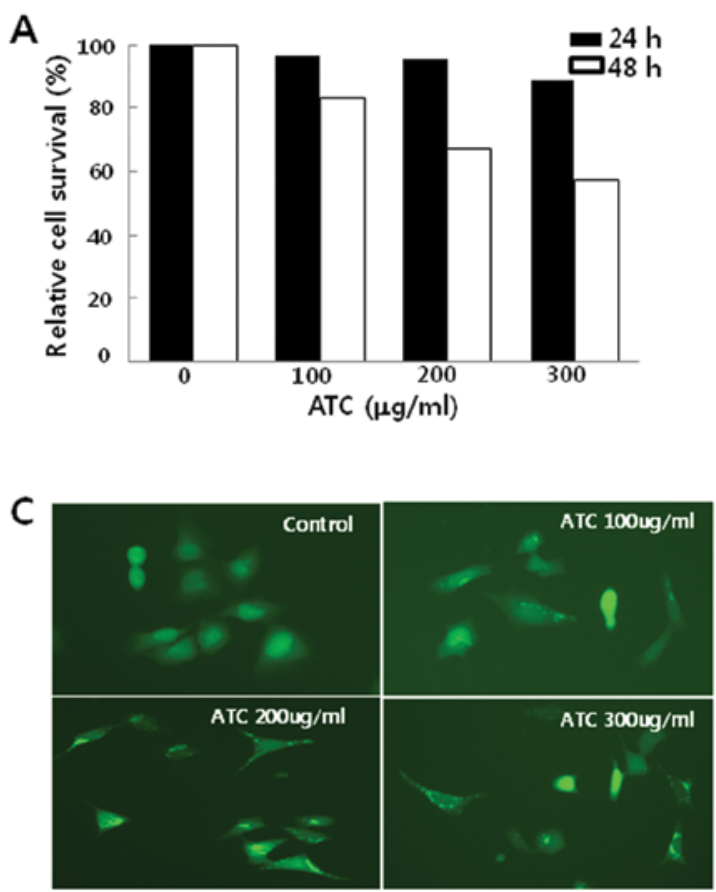

B
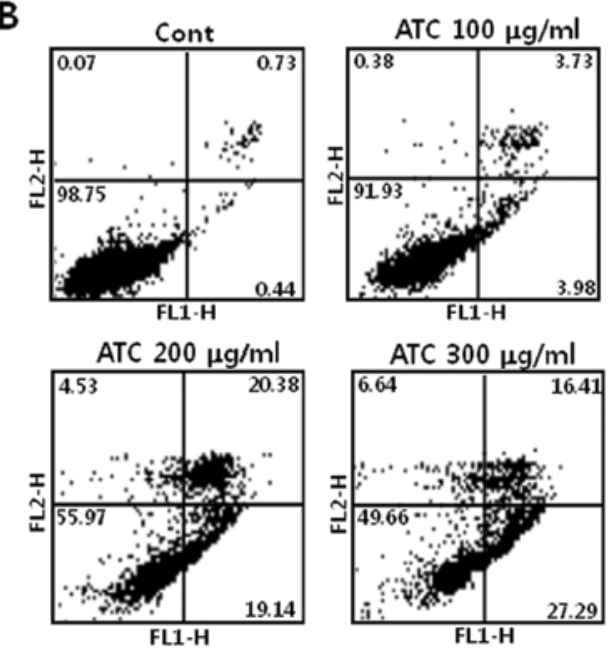

D

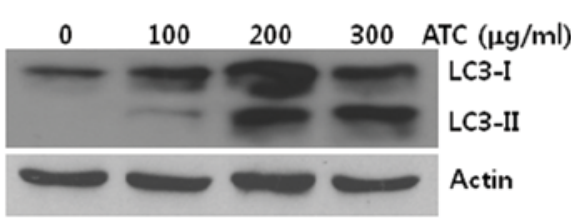

Figure 1. Induction of autophagy by ATCs. (A) U2OS cells were treated with the indicated concentrations of ATCs. After 24 and 48 h, MTT assays were performed and the data are presented as the relative cell survival (\%). (B) U2OS cells treated by ATCs at the indicated concentrations for $48 \mathrm{~h}$ were subjected to Annexin V/PI staining followed by flow cytometric analysis. (C) After U2OS-GFP-LC3 cells were treated with the indicated concentrations of ATCs for $6 \mathrm{~h}$, the cells were observed under a fluorescence microscope at a magnification $\mathrm{x} 40$. (D) U2OS cells treated the same as described in (C) were subjected to immunoblot analysis against LC3. Actin was used for the equal loading of proteins.

Transfection of small interfering RNA. Small interfering RNA against $A M P K \alpha 1$ (siAMPK) was purchased from SigmaAldrich Corp., and small interfering RNAs against $p 27^{K I P I}$ and $\mathrm{FOXO3a}$ were from Bioneer (Daejeon, Korea). Each siRNA was transfected into U2OS cells or U2OS-GFP-LC3 cells by the reverse transfection method using RNAiMAX reagent (Invitrogen) according to the manufacturer's protocol. The sequences of duplex siRNAs are as follows: siAMPK, 5'-CCCAUAUUAUUUGCGUGUA(dTdT)::5'UACACGCAAAUAAUAUGGG(dTdT); sip27 ${ }^{K I P l}, 5^{\prime}-\mathrm{CGA}$ CGAUUCUUCUACUCAA(dTdT)::5'-UUGAGUAGAAGA AUCGUCG(dTdT); siFOXO3a, 5'-GACGAUGAUGCGCCU CUCU(dTdT)::5'-AGAGAGGCGCAUCAUCGUC(dTdT).

Assessment of cell death. Cell death was examined by measuring sub-G1 cells and phosphatidylserine translocation. For measurement of sub-G1 cells, cells were fixed in icecold $70 \%$ ethanol for $2 \mathrm{~h}$ at $4{ }^{\circ} \mathrm{C}$ and stained with propidium iodide (PI; $0.2 \mathrm{mg} / \mathrm{ml})$, followed by flow cytometric analysis (FACSCalibur, BD Bioscience, San Jose, CA, USA). To determine phosphatidylserine translocation, the cells stained with PI and annexin V using ApoScan Kit (BioBud, Gyunggi-do, Korea) were analyzed using a previously described method (26).

Measurement of intracellular ATP levels. Intracellular ATP levels were measured using a CellTiter Glo Kit (Promega, Madison, WI, USA) following the manufacturer's instructions.

Statistical analysis. Statistical significances were evaluated using the one-way ANOVA test. The data represent three independent experiments performed in triplicate as the mean \pm SD.

\section{Results}

ATCs induce autophagy in U2OS cells. When human osteosarcoma U2OS cells were treated with ATCs, their growth was not inhibited in the first $24 \mathrm{~h}$. However, after $24 \mathrm{~h}$, ATCs suppressed the growth of U2OS cells associated with apoptosis (Fig. 1A and B), suggesting that ATCs activate the cell death program after $24 \mathrm{~h}$ in this cell line and autophagy may be induced before $24 \mathrm{~h}$. To investigate this further, we examined the autophagy by ATCs before $24 \mathrm{~h}$. For this end, we generated U2OS cells stably expressing GFP-LC3 (U2OSGFP-LC3) and first observed LC3 puncta formation in these cells. When U2OS-GFP-LC3 cells were treated with ATCs for $6 \mathrm{~h}$, it appeared that GFP-LC3 puncta were formed (Fig. 1C). Consistent with LC3 foci formation, the conversion of LC3-I to LC3-II occurred in both U2OS (Fig. 1D) and U2OS-GFP-LC3 cells (data not shown) in a dose-dependent manner, reaching a plateau level at concentrations of ATCs above $200 \mu \mathrm{g} / \mathrm{ml}$ of ATCs. These findings demonstrated that ATCs induced autophagy in U2OS cells prior to the activation of apoptosis.

ATC-induced autophagy is not prevented by inhibitors of $M A P K s$ or $A K T$. To identify the signal transduction pathway leading to autophagy in ATC-treated cells, we investigated the phosphorylation of signal transduction proteins which have been reported to be involved in ATC-induced apoptosis. As shown in Fig. 2A, the phosphorylation of ERK1/2, p38 MAPK, JNK, and AKT was increased by ATC treatment, suggesting that they are involved in this autophagy. However, neither the formation of GFP-LC3 puncta nor LC3-I conversion to LC3-II was prevented by any of the chemical inhibitors of AKT 

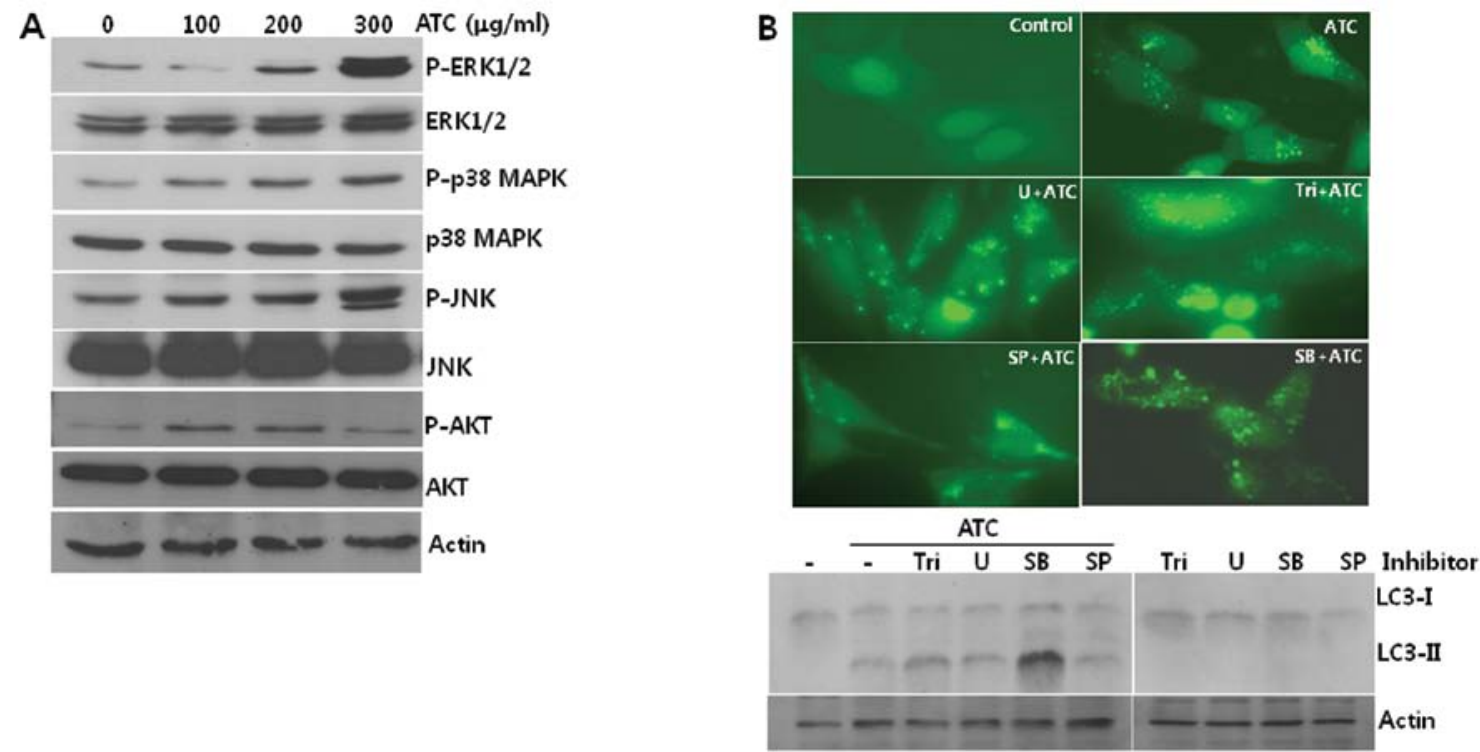

Figure 2. Activation of multiple protein kinases by ATCs. (A) Total cell lysates of U2OS cells treated with the indicated concentrations of ATCs were subjected to immunoblot analysis against the indicated proteins. (B) U2OS-GFP-LC3 (upper panel) or U2OS (lower panel) cells were pretreated with $20 \mu \mathrm{M}$ of each inhibitor against protein kinases for $1 \mathrm{~h}$ and incubated in the absence or presence of ATCs $(200 \mu \mathrm{g} / \mathrm{ml})$ for $6 \mathrm{~h}$. Cells were observed under a fluorescence microscope at a magnification x100 (upper panel) and total cell lysates were subjected to immunoblot analysis against LC3 (lower panel). Actin was used for the equal loading of proteins. Tri, triciribine; U, U0126; SB, SB203580; SP, SP600125.
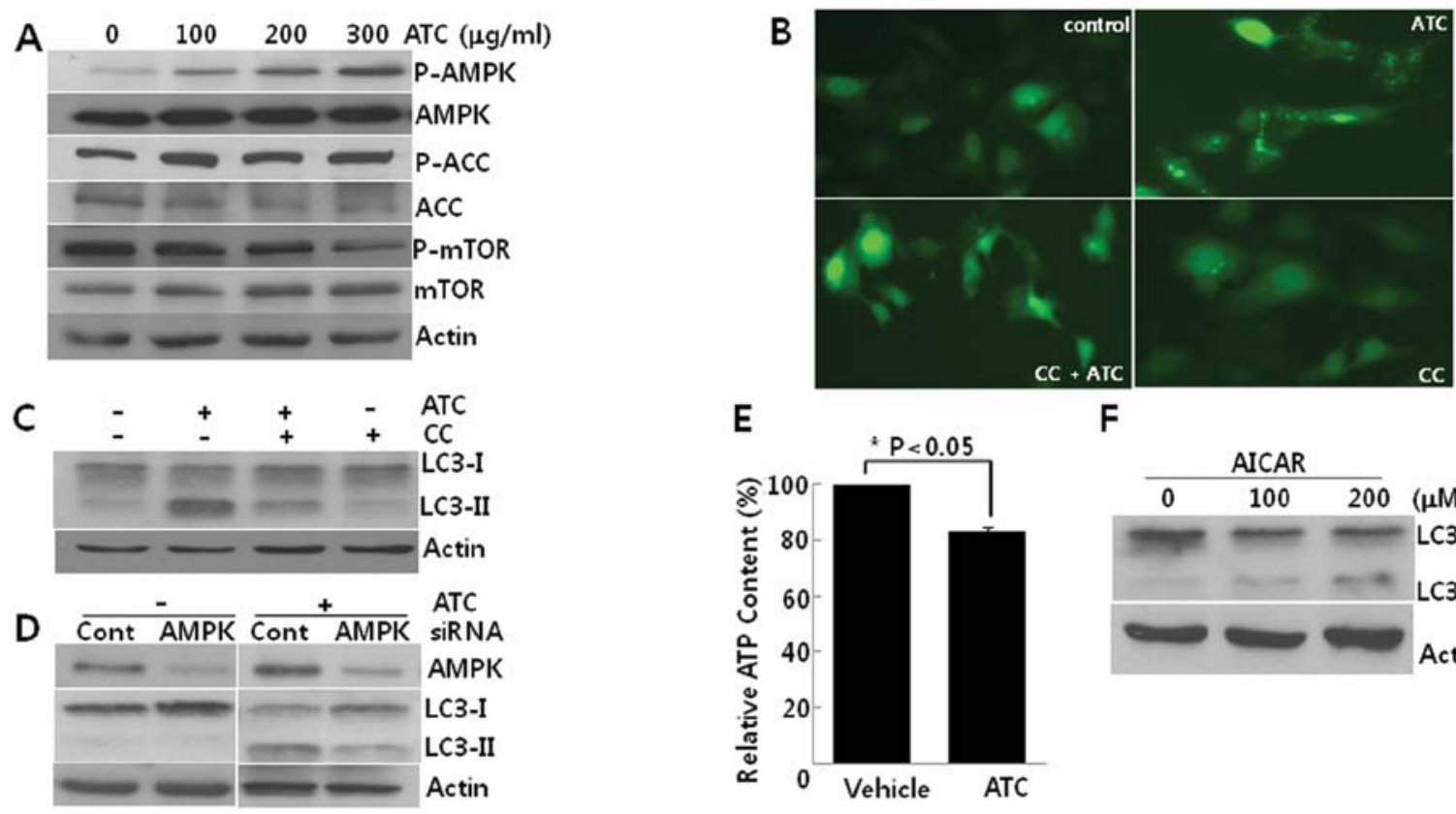

E

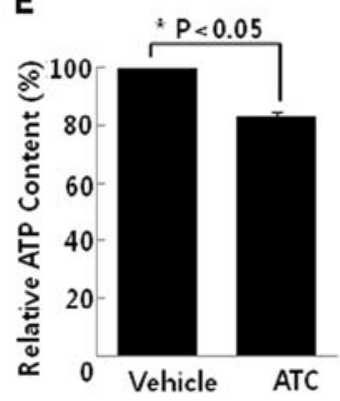

$\mathbf{F}$

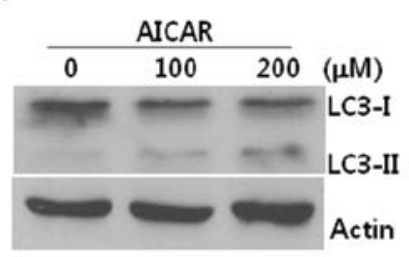

Figure 3. Prevention of ATC-induced autophagy by AMPK inhibition. (A) Lysates of U2OS cells incubated with the indicated concentrations of ATCs for $6 \mathrm{~h}$ were subjected to immunoblot analysis against the indicated proteins. (B) U2OS-GFP-LC3 or (C) U2OS cells pretreated with vehicle or CC (5 $\mu \mathrm{M})$ were incubated in the absence or presence of ATCs $(200 \mu \mathrm{g} / \mathrm{ml})$. After $6 \mathrm{~h}$, (B) cells were observed under a fluorescence microscope at a magnification x40 and (C) U2OS cell lysates were subjected to immunoblot analysis against LC3. (D) U2OS cells transfected with scrambled (control) siRNA or AMPK siRNA for $48 \mathrm{~h}$, were incubated in the presence or absence of ATCs $(200 \mu \mathrm{g} / \mathrm{ml})$ for a further $6 \mathrm{~h}$. Immunoblot analyses against the indicated proteins were performed (E) Intracellular ATP levels were measured in U2OS cells treated in triplicate with vehicle or ATCs $(200 \mu \mathrm{g} / \mathrm{ml})$ for $6 \mathrm{~h}$. The values represents the mean \pm SD of three independent experiments. (F) U2OS cells treated with the indicated concentrations of AICAR for $24 \mathrm{~h}$ were subjected to immunoblot analysis against LC3. Actin was used for the equal loading of proteins.

(Triciribine, Tri), MEK (U0126, U), p38 MAPK (SB203580, SB), and JNK (SP600125, SP) (Fig. 2B). These findings led us to speculate that pathways other than AKT and MAPKs including p38 MAPK, ERK, and JNK may contribute to the induction of autophagy by ATCs.
ATC-induced autophagy is prevented by AMPK inhibition. We then explored the activation of AMPK in ATC-treated cells. While the phosphorylation of mTOR was decreased, the phosphorylation of both AMPK $\alpha 1$ and its substrate, acetyl CoA carboxylase (ACC) increased according to concentrations 
A

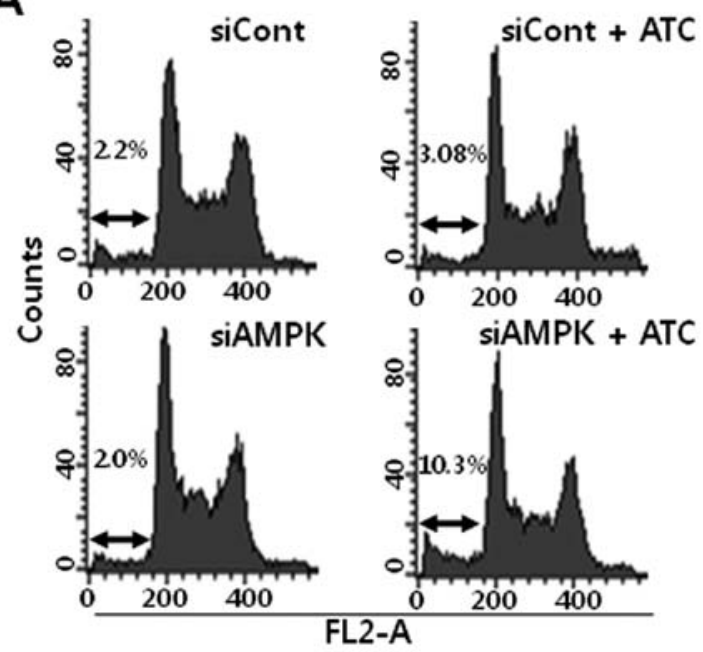

B

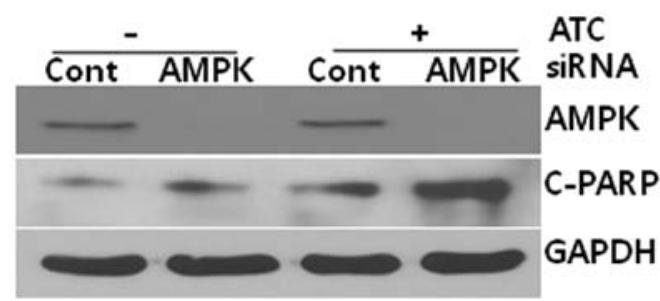

Figure 4. Increase of ATC-induced apoptosis by AMPK-knockdown. U2OS cells transfected by siRNA against $A M P K \alpha 1$ or scrambled siRNA as a negative control for $48 \mathrm{~h}$ were incubated in the absence or presence of ATCs $(200 \mu \mathrm{g} / \mathrm{ml})$ for a further $6 \mathrm{~h}$. Cells were then processed for (A) cell cycle analysis using FACSCalibur, and for (B) immunoblot analysis against cleaved poly(ADPribose) polymerase-1 (p25, C-PARP). GAPDH was used as a control. of ATCs, indicating that AMPK is activated in this autophagy (Fig. 3A). Pretreatment with CC, an inhibitor of AMPK, reduced both the GFP-LC3 puncta formation (Fig. 3B) and the conversion of LC3-I to LC3-II (Fig. 3C). Consistent with the effect of CC, the knockdown of AMPKa1 using siRNA against $A M P K \alpha 1$ also suppressed the conversion of LC3-I to LC3-II (Fig. 3D). Furthermore, ATCs diminished intracellular ATP levels (Fig. 3E) and AICAR, an analog of AMP, also induced autophagy in U2OS cells (Fig. 3F). Collectively, these findings suggest that ATC-induced autophagy is dependent on AMPK activation which may be due to the depletion of ATP.

AMPK inhibition enhances ATC-induced apoptosis. Based on the report that autophagy inhibition augmented the ATC-induced apoptosis in hepatocellular carcinoma cells (13), we analyzed the effect of AMPK on apoptosis in ATC-treated U2OS cells. As shown in Fig. 4, while ATCs induced a subtle apoptosis at $6 \mathrm{~h}$ in $\mathrm{U} 2 \mathrm{OS}$ cells that had been transfected with control siRNA, ATCs dramatically induced apoptotic features such as accumulation of hypo-diploidic cells (Fig. 4A) and poly(ADP-ribose) polymerase-1 (PARP) cleavage (Fig. 4B) in $A M P K \alpha 1$ siRNA-transfected U2OS cells. Thus, these data suggest that ATC-activated AMPK protects U2OS cells from apoptosis.

Differential effects of $p 27^{K I P I}$ and FOXO3a on ATC-induced autophagy. To investigate the downstream targets of AMPK that play a role in the induction of autophagy, we attempted the transfection of siRNAs against $U L K 1, \mathrm{p} 27^{\mathrm{KIP} 1}$, and FOXO3a
A
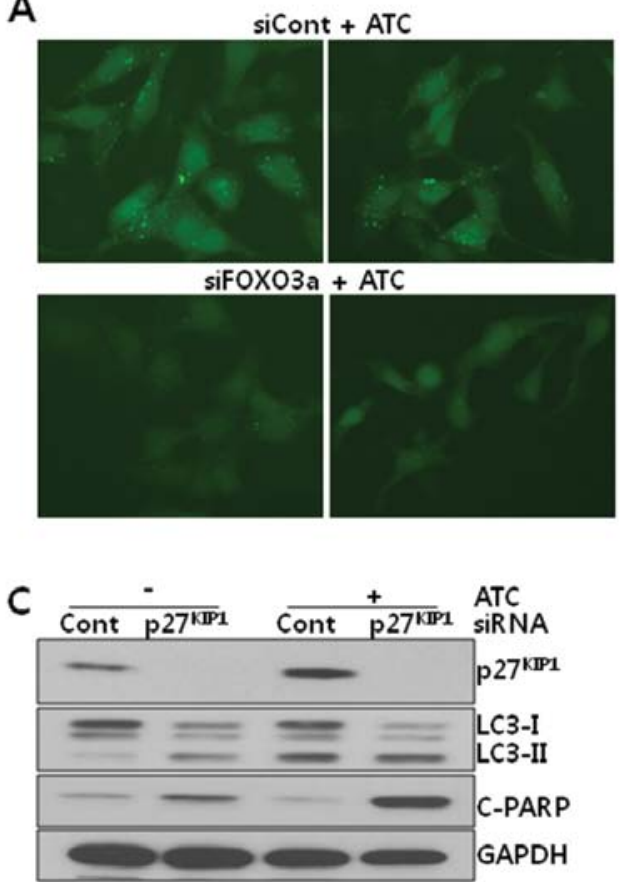

B

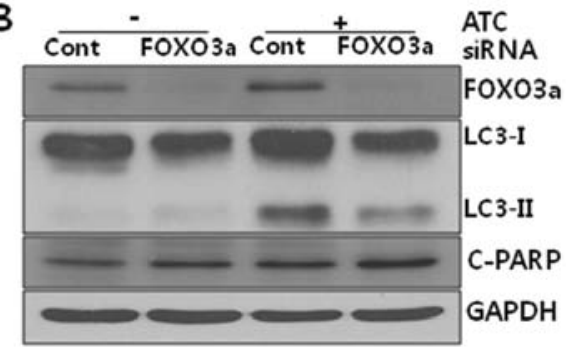

D

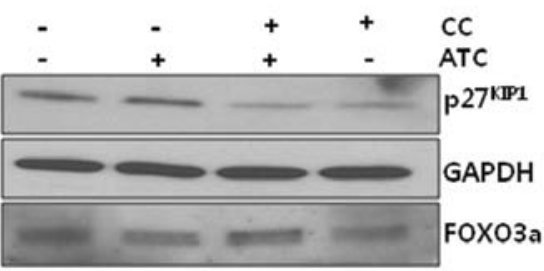

E

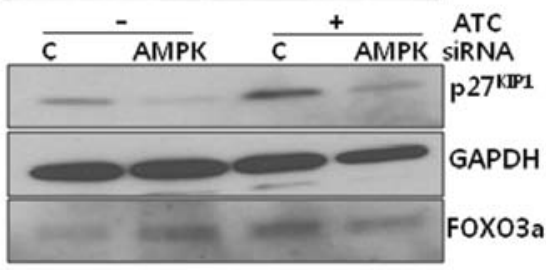

Figure 5. The effect of siRNA against FOXO3a or $p 27^{K I P I}$ on the ATC-induced autophagy. U2OS-GFP-LC3 or U2OS cells transfected by siRNA against scrambled (control) siRNA or FOXO3a for $48 \mathrm{~h}$ were incubated with ATCs $(200 \mu \mathrm{g} / \mathrm{ml})$ for a further $6 \mathrm{~h}$. (A) U2OS-GFP-LC3 cells were then observed under a fluorescence microscope at a magnification x100. (B) U2OS cells were then subjected to immunoblot analysis. (C) U2OS cells transfected by scrambled (control) siRNA or $p 27^{K I P}$ siRNA for $48 \mathrm{~h}$ were incubated with ATCs for a further $6 \mathrm{~h}$. Cells were then subjected to immunoblot analyses. (D) U2OS cells pretreated with CC $(5 \mu \mathrm{M})$ for $1 \mathrm{~h}$ were incubated in the presence of vehicle or ATCs $(200 \mu \mathrm{g} / \mathrm{ml})$ for a further $6 \mathrm{~h}$, followed by immunoblot analyses. (E) U2OS cells transfected with scrambled (control) siRNA or AMPKal siRNA for $48 \mathrm{~h}$ were treated with vehicle or ATCs (200 $\mu \mathrm{g} / \mathrm{ml})$ for a further $6 \mathrm{~h}$, followed by immunoblot analyses. GAPDH was used for the equal loading of proteins. C-PARP, p25 cleaved-poly(ADP-ribose) polymerase-1. 
which have been demonstrated to be autophagy modulators under AMPK. The knockdown of ULK1 by ULK1 siRNA did not exert an inhibitory effect on either ATC-induced autophagy or apoptosis, ruling out the involvement of ULK1 in this autophagy (data not shown). As shown in Fig. 5, both ATC-induced LC3 puncta formation (Fig. 5A) and the conversion of LC3-I to LC3-II (Fig. 5B) were reduced, but ATC-induced PARP cleavage was not altered by FOXO3a knockdown, suggesting that FOXO3a may be responsible for the induction of autophagy induction under active AMPK. In contrast to the effect of FOXO3a siRNA, $p 27^{K I P I}$ siRNA potentiated ATC-induced PARP cleavage but had no effect on LC3-II levels (Fig. 5C), suggesting that $\mathrm{p} 27^{\mathrm{KIPl}}$ may inhibit ATC-induced apoptosis, but has no effect on autophagy. Thus, it can be suggested that AMPK activated by ATCs may induce autophagy via FOXO3a and inhibit apoptosis via p2 $7^{\mathrm{KIP} 1}$.

\section{Discussion}

The findings reported herein indicate that ATCs induce autophagy in human osteosarcoma U2OS cells, accompanied by the activation of MAPKs and AMPK. By analyzing the effects of chemical inhibitors and siRNAs against MAPKs and AMPK on ATC-induced autophagy, the findings suggest that AMPK plays a critical role in this autophagy. However, how ATCs specifically activate AMPK, remains unknown. It has been reported that phytochemicals such as galegine, berberine, as well as resveratrol activate AMPK in an AMP-dependent manner (27). ATCs also reduced ATP levels in this cell line and AICAR, an analog of AMP, induced autophagy (Fig. 3E and $\mathrm{F}$ ), indicating that AMP/LKB1-dependent AMPK activation results from ATC treatment. However, LKB1 was not phosphorylated by ATCs and AMPK was still phosphorylated in U2OS cells that had been transfected with LKB1 siRNA (data not shown). In addition, ATC-induced AMPK phosphorylation was not altered by BAPTA-AM, a calcium chelator, CaMKK siRNA, TAKI siRNA or ROS scavengers (data not shown). Collectively, it can be assumed that ATCs may enrich intracellular AMP levels which induce the allosteric activation of AMPK without the action of LKB1 or inhibit the dephosphorylation of AMPK that was phosphorylated by unidentified enzymes other than LKB1. However, as it is known that the allosteric activation of AMPK by AMP is induced in AMPK phosphorylated by basal LKB1 activity, this assumption leaves many questions to be resolved. Considering the numerous studies reporting that AMPK-activating compounds including phytochemicals inhibit mitochondrial respiratory complexes to reduce synthesis of ATP, we speculate that ATCs might contain two major features for activating AMPK: one is to reduce ATP synthesis possibly via the inhibition of mitochondrial respiratory complexes and the other is to induce the basal phosphorylation of AMPK or the allosteric activation of AMPK in conjunction with AMP even in the absence of LKB1 activity.

A recent study showed that AMPK phosphorylates ULK1 to initiate autophagy (18). In the present model, ATC-induced AMPK phosphorylation was accompanied by the dephosphorylation of mTOR (Fig. 3) and rapamycin, an inhibitor of mTOR, induced autophagy (data not shown). Moreover, ATCs induced autophagy in ULK1-knocked down cells to a comparable extent as in control siRNA-transfected U2OS cells

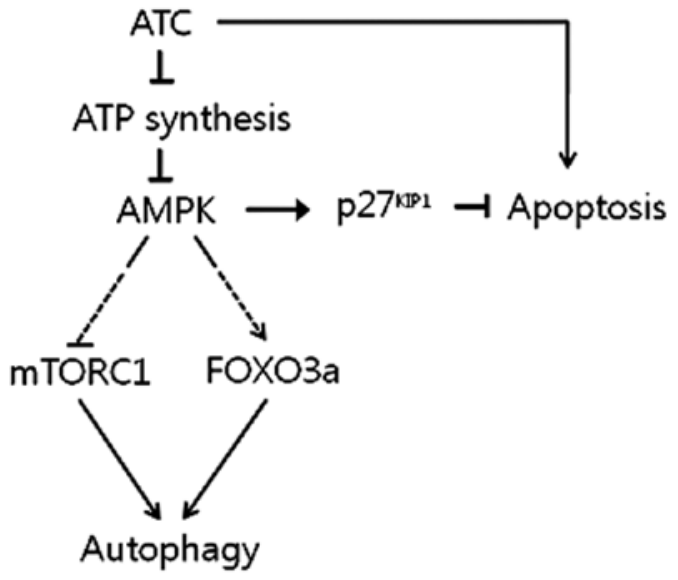

Figure 6. Schematic diagram representing the proposed mechanism of ATCinduced autophagy in U2OS cells.

(data not shown), suggesting that AMPK-induced mTORC1 inhibition may play a more important role in ATC-induced autophagy than does ULK1 activation. In addition to the mTOR and ULK1 pathways, AMPK modulates downstream target proteins such as $\mathrm{p} 27^{\mathrm{KIPl}}$ and FOXO3a to induce autophagy. P27 ${ }^{\mathrm{KIP} 1}$, an inhibitor of CDK1, which can be phosphorylated by AMPK and can be induced transcriptionally by FOXO3a as well, was reported to induce autophagy but inhibit apoptosis (19), suggesting that it is a strong molecular target of ATC-activated AMPK. Different from these reports, in our model, it appeared that $\mathrm{p} 27^{\mathrm{KIP} 1}$ inhibited apoptosis but was not involved in autophagy, whereas FOXO3a contributed to the induction of autophagy but not apoptosis. Therefore, it can be concluded that AMPK activated by ATC-inhibited ATP synthesis induces autophagy by activating FOXO3a while suppressing mTORC1, and inhibits ATC-induced apoptosis via the upregulation of $\mathrm{p} 27^{\mathrm{KIP} 1}$, as summarized in Fig. 6. To verify this, however, the mechanism by which $\mathrm{p} 27^{\mathrm{KIP} 1}$ and FOXO3a are regulated by ATCs remains to be clarified. As shown in Fig. 5C-E, p2 $7^{\mathrm{KIP1}}$ protein levels were upregulated by ATC treatment, which returned to basal levels in U2OS cells that had been pretreated with compound C (CC) or transfected with $A M P K \alpha 1$ siRNA before ATC treatment, suggesting that p2 $7^{\mathrm{KIP} 1}$ may be stabilized by active AMPK. In contrast to p2 $7^{\mathrm{KIP} 1}$, FOXO3a protein levels were not consistently altered by ATCs and the phosphorylation of FOXO3a-Ser413, a site known to be phosphorylated by AMPK, was not detected either (data not shown), suggesting that the phosphorylation of Ser or Thr residues other than Ser413 by AMPK or an indirect effect of AMPK on FOXO3a induces autophagy.

The role of AMPK in cancer cells is known to be varied. AMPK can activate p53 by phosphorylating p53-Ser15 to induce cell cycle arrest and apoptosis $(28,29)$. For example, a combined treatment with metformin and 2-deoxyguanosine, as well as vincristine, an anticancer agent, induced apoptosis in prostate and melanoma cells, respectively, by activating AMPK $(30,31)$. Contrary to these reports, AMPK inhibition resulted in the sensitization of cancer cells to apoptosis by anticancer drugs such as cisplatin as well as metabolic stress such as hypoxia $(32,33)$. This variable effect of AMPK on cell death may be due to both types of stresses and cellular 
context. It was recently reported that autophagy inhibition can enhance the cytotoxicity of ATCs in hepatoma cells (13), which may be consistent with the data in our study showing that AMPK and $227^{\mathrm{KIP} 1}$ siRNAs enhanced ATC-induced apoptosis (Figs. 4 and 5C). Therefore, AMPK activation by ATCs may contribute to the cancer cell survival via the induction of autophagy.

In non-transformed cells, autophagy serves to preserve genomic integrity, thereby preventing malignant transformations (34). This autophagy-inducing activity of ATCs could explain the chemopreventive effect of ATCs reported in the esophagus and the colon $(11,12)$, although the autophagyinducing activity of ATCs in non-transformed cells remains to be determined. A metabolic shift to aerobic glycolysis has generally been regarded as a driving force for carcinogenesis $(35,36)$, and dysregulated metabolic homeostasis and obesity have been reported to increase the risk of certain types of cancer, such as breast cancer (37). Since AMPK is a central regulator of metabolism and preserves metabolic homeostasis, AMPK could prevent cancer formation under conditions of metabolic distress. Consistent with this assumption, metformin was reported to inhibit the formation of pre-neoplastic lesions in the colon and showed chemopreventive activity against breast cancer associated with obesity (38). Therefore, even if ATCs do not induce autophagy in non-transformed cells, only the AMPK-activating effect of ATCs could contribute to chemopreventive effects against cancer.

Autophagy can prevent the induction of apoptosis and, instead, initiate necrotic cell death in cancer cells in vivo, accompanied by an inflammatory response, which can contribute to the invasion and metastasis of cancer cells $(39,40)$. This autophagy-inducing activity of ATCs could dampen the anticancer effect of ATCs, thereby constituting a negative feedback loop of ATC-induced apoptosis.

Collectively, it could be concluded that while ATCs may activate AMPK and, thus, prevent carcinogenesis in nontransformed cells by activating autophagy and preserving metabolic homeostasis, autophagy in cancer cells may diminish the apoptosis-inducing activity of ATCs. Therefore, to develop ATCs for use as efficient anticancer therapy or as a chemopreventive agent against cancer, the mechanism underlying the autophagy-inducing activity of ATCs as well as ATC-induced apoptosis needs to be thoroughly examined to determine whether it is possible to modulate genes to enhance the anticancer effects of ATCs.

\section{Acknowledgements}

This study was supported by the Biogreen 21 Program (code no. PJ007186), Rural Development Administration Korea. Part of this study was also supported by the Basic Science Research Program through the National Research Foundation of Korea (NRF) funded by the Ministry of Education, Science and Technology (2011-0027115).

\section{References}

1. Yeh CT and Yen GC: Induction of apoptosis by the anthocyanidins through regulation of Bcl-2 gene and activation of c-Jun $\mathrm{N}$-terminal kinase cascade in hepatoma cells. J Agric Food Chem 53: 1740-1749, 2005
2. Shin DY, Lee WS, Lu JN, et al: Induction of apoptosis in human colon cancer HCT-116 cells by anthocyanins through suppression of Akt and activation of p38-MAPK. Int J Oncol 35: 1499-1504, 2009.

3. Shih PH, Yeh CT and Yen GC: Effects of anthocyanidin on the inhibition of proliferation and induction of apoptosis in human gastric adenocarcinoma cells. Food Chem Toxicol 43: 1557-1566, 2005.

4. Lee SH, Park SM and Park SM: Induction of apoptosis in human leukemia U937 cells by anthocyanins through down-regulation of Bcl-2 and activation of caspases. Int J Oncol 34: 1077-1083, 2009.

5. Reddivari L, Vanamala J, Chintharlapalli S, et al: Anthocyanin fraction from potato extracts is cytotoxic to prostate cancer cells through activation of caspase-dependent and caspase-independent pathways. Carcinogenesis 28: 2227-2235, 2007.

6. Hou DX, Fujii M, Terahara N and Yoshimoto M: Molecular mechanisms behind the chemopreventive effects of anthocyanins. J Biomed Biotechnol 5: 321-325, 2004.

7. Tsoyi K, Park HB, Kim YM, et al: Anthocyanins from black soybean seed coats inhibit UVB-induced inflammatory cylooxygenase- 2 gene expression and $\mathrm{PGE}_{2}$ production through regulation of the nuclear factor- $\mathrm{\kappa} \mathrm{B}$ and phosphatidylinositol 3-kinase/Akt pathway. J Agric Food Chem 56: 8969-8974, 2008.

8. Sautebin L, Rossi A, Serraino I, et al: Effect of anthocyanins contained in a blackberry extract on the circulatory failure and multiple organ dysfunction caused by endotoxin in the rat. Planta Med 70: 745-752, 2004.

9. Shin DY, Lu JN, Kim GY, et al: Anti-invasive activities of anthocyanins through modulation of tight junctions and suppression of matrix metalloproteinase activities in HCT-116 human colon carcinoma cells. Oncol Rep 25: 567-572, 2011.

10. Matsubara K, Kaneyuki T, Miyake T and Mori M: Antiangiogenic activity of nasunin, an antioxidant anthocyanin, in eggplant peels. J Agric Food Chem 53: 6272-6275, 2005.

11. Wang LS, Hecht SS, Carmella SG, et al: Anthocyanins in black raspberries prevent esophageal tumors in rats. Cancer Prev Res 2: 84-93, 2009.

12. Thomasset S, Berry DP, Cai H, et al: Pilot study of oral anthocyanins for colorectal cancer chemoprevention. Cancer Prev Res 2: 625-633, 2009.

13. Longo L, Platini F, Scardino A, Alabiso O, Vasapollo G and Tessitore L: Autophagy inhibition enhances anthocyanininduced apoptosis in hepatocellular carcinoma. Mol Cancer Ther 7: 2476-2485, 2008.

14. Kim YK, Yoon HH, Lee YD, et al: Anthocyanin extracts from black soybean (Glycine max L.) protect human glial cells against oxygen-glucose deprivation by promoting autophagy. Biomol Ther 20: 68-74, 2012.

15. Hardie DG: AMP-activated protein kinase: an energy sensor that regulates all aspects of cell function. Genes Dev 25: 1895-1908, 2011.

16. Yun $\mathrm{H}$ and $\mathrm{Ha}$ J: AMP-activated protein kinase modulators: a patent review (2006-2010). Expert Opin Ther Pat 21: 983-1005, 2011.

17. Alers S, Löffler AS, Wesselborg S and Stork B: Role of AMPKmTOR-Ulk1/2 in the regulation of autophagy: cross talk, shortcuts, and feedbacks. Mol Cell Biol 32: 2-11, 2012.

18. Egan DF, Shackelford DB, Mihaylova MM, et al: Phosphorylation of ULK1 (hATG1) by AMP-activated protein kinase connects energy sensing to mitophagy. Science 331: 456-461, 2011.

19. Liang J, Shao SH, Xu ZX, et al: The energy sensing LKB1-AMPK pathway regulates $\mathrm{p} 27^{\mathrm{KIPl}}$ phosphorylation mediating the decision to enter autophagy or apoptosis. Nat Cell Biol 9: 218-224, 2007.

20. Greer EL, Oskoui PR, Banko MR, et al: The energy sensor AMP-activated protein kinase directly regulates the mammalian FOXO3 transcription factor. J Biol Chem 282: 30107-30119, 2007.

21. Shi J, Zhang L, Shen A, et al: Clinical and biological significance of forkhead class box $\mathrm{O} 3 \mathrm{a}$ expression in glioma: mediation of glioma malignancy by transcriptional regulation of $\mathrm{p} 27^{\mathrm{KIP} 1}$. J Neurooncol 98: 57-69, 2010.

22. Gopinath S, Malla RR, Gondi CS, et al: Co-depletion of cathepsin B and UPAR induces G0/G1 arrest in glioma via FOXO3a mediated p27 upregulation. PLoS One 5: e11668, 2010.

23. Lee YK, Lee WS, Kim GS and Park OJ: Anthocyanins are novel AMPK $\alpha 1$ stimulators that suppress tumor growth by inhibiting mTOR phosphorylation. Oncol Rep 24: 1471-1477, 2010.

24. Jang H, Ha US, Kim SJ, Yoon BI, Han DS, Yuk SM and Kim SW: Anthocyanin extracted from black soybean reduces prostate weight and promotes apoptosis in the prostatic hyperplasiainduced rat model. J Agric Food Chem 58: 12686-12691, 2010. 
25. Lee JH, Kang NS, Shin SO, et al: Characterization of anthocyanin in the black soybean (Glycine max L.) by HPLC-DAD-ESI/MS analysis. Food Chem 112: 226-231, 2009.

26. Jang JY, Kim MK, Jeon YK, Joung YK, Park KD and Kim CW: Adenovirus adenine nucleotide translocator-2 shRNA effectively induces apoptosis and enhances chemosensitivity by the downregulation of ABCG2 in breast cancer stem-like cells. Exp Mol Med 44: 251-259, 2012

27. Simon A. Hawley, Fiona A. Ross, Cyrille Chevtzoff, et al: Use of cells expressing $\gamma$ subunit variants to identify diverse mechanisms of AMPK activation. Cell Metab 11: 554-565, 2010.

28. Jones RG, Plas DR, Kubek S, et al: AMP-activated protein kinase induces a p53-dependent metabolic checkpoint. Mol Cell 18 283-293, 2005

29. Imamura K, Ogura T, Kishimoto A, Kaminishi $M$ and Esumi $H$ : Cell cycle regulation via 53 phosphorylation by a 5'-AMP activated protein kinase activator, 5-aminoimidazole-4-carboxamide-1- $\beta$ D-ribofuranoside, in a human hepatocellular carcinoma cell line. Biochem Biophys Res Commun 287: 562-567, 2001.

30. Ben Sahra I, Laurent K, Giuliano S, et al: Targeting cancer cell metabolism: The combination of metformin and 2-deoxyglucose induces p53-dependent apoptosis in prostate cancer cells. Cancer Res 70: 2465-2475, 2010.

31. Chen MB, Shen WX, Yang Y, Wu XY, Gu JH and Lu PH: Activation of AMP-activated protein kinase is involved in vincristine-induced cell apoptosis in B16 melanoma cell. J Cell Physiol 226: 1915-1925, 2011.
32. Kim HS, Hwang JT, Yun H, et al: Inhibition of AMP-activated protein kinase sensitizes cancer cells to cisplatin-induced apoptosis via hyper-induction of p53. J Biol Chem 283: 3731-3742, 2008.

33. Chhipa RR, Wu Y and Ip C: AMPK-mediated autophagy is a survival mechanism in androgen-dependent prostate cancer cells subjected to androgen deprivation and hypoxia. Cell Signal 23 1466-1472, 2011.

34. Karantza-Wadsworth V, Patel S, Kravchuk O, et al: Autophagy mitigates metabolic stress and genome damage in mammary tumorigenesis. Genes Dev 21: 1621-1635, 2007.

35. Kim JW and Dang CV: Cancer's molecular sweet tooth and the Warburg effect. Cancer Res 66: 8927-8930, 2006.

36. Levine AJ and Puzio-Kuter AM: The control of the metabolic switch in cancers by oncogenes and tumor suppressor genes. Science 330: 1340-1344, 2010.

37. Cleary MP and Grossman ME: Minireview: obesity and breast cancer - the estrogen connection. Endocrinology 150: 2537-2542, 2009.

38. Goodwin PJ and Stambolic V: Obesity and insulin resistance in breast cancer - Chemoprevention strategies with a focus on metformin. Breast S3: S31-S35, 2011.

39. Mathew R, Karantza-Wadsworth $\mathrm{V}$ and White E: Role of autophagy in cancer. Nat Rev Cancer 7: 961-967, 2007.

40. Eisenberg-Lerner A, Bialik S, Simon HU and Kimchi A: Life and death partners: apoptosis, autophagy and the cross-talk between them. Cell Death Diff 16: 966-975, 2009. 